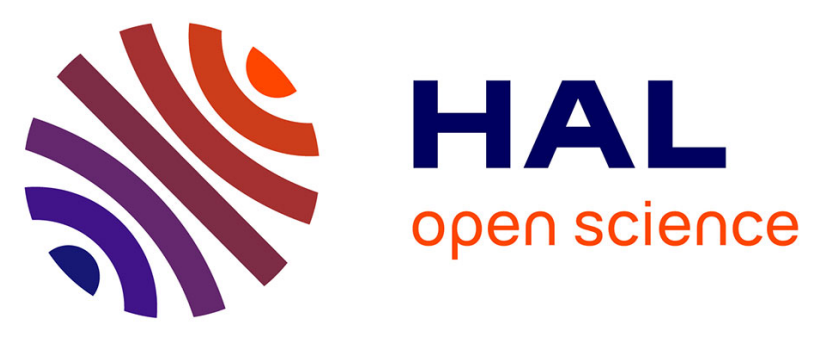

\title{
Dark current analysis of Quantum Cascade Detectors by Magneto-Resistance measurements
}

\author{
Aurore Gomez, Nicolas Péré-Laperne, Louis-Anne de Vaulchier, Cédric \\ Koeniguer, Angela Vasanelli, Alexandru Nedelcu, Xavier Marcadet, Yves \\ Guldner, Vincent Berger
}

\section{To cite this version:}

Aurore Gomez, Nicolas Péré-Laperne, Louis-Anne de Vaulchier, Cédric Koeniguer, Angela Vasanelli, et al.. Dark current analysis of Quantum Cascade Detectors by Magneto-Resistance measurements. Physical Review B: Condensed Matter and Materials Physics (1998-2015), 2008, 77, pp.085307. hal00193979

\section{HAL Id: hal-00193979 \\ https://hal.science/hal-00193979}

Submitted on 5 Dec 2007

HAL is a multi-disciplinary open access archive for the deposit and dissemination of scientific research documents, whether they are published or not. The documents may come from teaching and research institutions in France or abroad, or from public or private research centers.
L'archive ouverte pluridisciplinaire $\mathbf{H A L}$, est destinée au dépôt et à la diffusion de documents scientifiques de niveau recherche, publiés ou non, émanant des établissements d'enseignement et de recherche français ou étrangers, des laboratoires publics ou privés. 


\title{
Dark current analysis of Quantum Cascade Detectors by Magneto-Resistance measurements
}

\author{
Aurore Gomez, ${ }^{1,2}$ Nicolas Péré-Laperne,${ }^{3}$ Louis-Anne de Vaulchier, ${ }^{3}$ Cédric Koeniguer, ${ }^{1}$ Angela \\ Vasanelli, ${ }^{1}$ Alexandro Nedelcu, ${ }^{2}$ Xavier Marcadet,${ }^{2}$ Yves Guldner, ${ }^{3}$ and Vincent Berger ${ }^{1,2, *}$ \\ ${ }^{1}$ Laboratoire Matériaux et Phénomènes Quantiques, \\ CNRS, Université Denis Diderot Paris 7, \\ Batiment Condorcet, 75205 Paris Cedex 13, France \\ ${ }^{2}$ Alcatel-Thales 3-5 lab, Route départementale 128, 91767 Palaiseau Cedex, France \\ ${ }^{3}$ Laboratoire Pierre Aigrain, CNRS, Ecole Normale Supérieure, \\ 24 rue Lhomond, 75231 Paris Cedex 05, France
}

(Dated: December 5, 2007)

\begin{abstract}
Magneto-transport experiments have been performed on Quantum Cascade Detectors. These experiments lead to the identification of the different electronic transitions from subbands in one cascade period to subbands in the following one. These transitions contribute to the total current flowing through the structure in the absence of illumination. This dark current is well described within a simple model based on the sum of diffusion events from one cascade to the next one through optical phonon mediated transitions. For the first time, the optical and electronic properties of such a complex heterostructure can be fully predicted without any other adjustable parameter than the doping density. This opens the way to a full quantum design of an infrared detector, in contrast with the phenomenological optimization of structures usually performed in this field.
\end{abstract}




\section{INTRODUCTION}

The electronic transport properties of complex heterostructures are the subject of an increasing number of theoretical studies. The Quantum Cascade Detector (QCD) ${ }^{1-4}$ recently proposed and realized as a photovoltaic version of Quantum Well Infrared Photodetectors (QWIPs) ${ }^{5}$, is a typical example of such a mesoscopic and complex structure. A period contains an "active region" dedicated to the absorption of infrared photons from the ground level to the upper levels of the structure ( $E_{1^{\prime}}$ to $E_{7}$ and $E_{8}$ as shown in figure 1). A following part is optimized for the electrons transfer through the period. The QCD structure is designed to generate an electronic displacement under illumination through a cascade of quantum levels without the need of an applied bias voltage (see figure 1). Owing to their photovoltaic behavior, QCDs can work with higher doping levels than QWIPs and therefore achieve higher quantum efficiencies, longer integration times. QCDs can also be used at low voltage and present lower dark currents. To optimize the performances of QCDs, a deep understanding of the transport in these complex heterostructures is required. In a photovoltaic detector, the zero voltage resistance (usually expressed as $R_{0} A$ where $A$ is the area of the device) is one of the relevant figures of merit to characterize the dark current measured in the absence of illumination. $R_{0} A$ can usually be described with an activation energy $E_{a}$, corresponding to the energy of the transition responsible for electron transfer from one contact to the other. In the case of QCDs, this picture has to be revisited because the dark current generally involves several diagonal transitions from one cascade to the next. In order to reveal all these parallel contributions, magneto-transport measurements as a function of temperature has been performed (reported in part III) allowing a clear identification of the different electronic paths that contribute significantly to the dark current. This technique acts as a very effective tool for checking that the quantum structure of the detector has been well designed. To address this complexity, a model of the electronic transport has been developed. This model (presented in part IV) takes into account all the possible electronic paths through the structure and contribute as parallel channels to the total current.

Let us first summarize the different rules for the design of a QCD structure, which results in a trade off. For a high photoresponse, the first requirement is to achieve a large optical matrix element between the fundamental level $\left(E_{1^{\prime}}\right)$ and the upper levels of the active region $\left(E_{7 / 8}\right.$ in our structure, where $E_{7 / 8}$ stands for " $E_{7}$ and $E_{8}$ "). A second important factor is to achieve a 
good extraction of the excited electrons from the upper levels to the right of the structure, down through the cascade $\left(E_{7 / 8}\right.$ down to $\left.E_{j}, j \leq 6\right)$. This implies both a sufficient density of subbands in the cascade and a good coupling between the different levels (through the electron-phonon interaction). Further, in the photovoltaic mode the noise of the detector is given by the Johnson noise, $4 k_{B} T / R_{0}$. A third requirement is therefore to realize a high resistance device. As will be shown in detail later, the total conductance of a quantum cascade structure can be described as the sum of the partial conductance of different paths in parallel, each of these paths being an intersubband phonon-mediated transition from one subband in a cascade " $A$ " $\left(E_{i}, i \in\left[1^{\prime}-8^{\prime}\right]\right)$ to another subband in the next cascade " $B$ " $\left(E_{j}, j \in[1-8]\right)$. The total current density can then be expressed as:

$$
J=e \sum_{i \in A} \sum_{j \in B}\left(G_{i j}(V)-G_{j i}(V)\right)
$$

where e is the electronic charge and $G_{i j}$ is the global electronic transfer rate from subband $i$ to subband $j$, which depends on the applied bias $V$ and can be calculated with the introduction of the electron-phonon Hamiltonian. To lower the conductance, it is necessary to "separate" two successive cascades (thus lowering the $G_{1^{\prime} j}$ ). This separation is nevertheless in contradiction with the two requirements for a high photoresponse i.e. a good optical coupling $E_{1^{\prime}} \rightarrow E_{7 / 8}$ and a good relaxation coupling $E_{7 / 8} \rightarrow E_{j}(j \leq 6)$. Intuitively, a good trade-off is found when the dark current is governed by the optical transition $E_{1^{\prime}} \rightarrow E_{7 / 8}$. An old principle of infrared detection is found: it is desirable to have a dark transport with an activation energy as close as possible to the optical transition. According to the $G_{i j}$ formalism, the condition can be expressed simply by stating that $G_{1^{\prime} j}$ has to be of the same order than $G_{1^{\prime} 7}$ and $G_{1^{\prime} 8}$. To calculate the different transitions rates, an accurate model is necessary. In addition, experimental results of the different cross transitions are also necessary to validate the model and the whole design process. In the following, we will show that magneto-transport experiments are a key tool for this purpose.

\section{QCD STRUCTURE}

The QCD under study is a GaAs/AlGaAs heterostructure with a detection wavelength of $8 \mu \mathrm{m}$. It consists of 40 identical periods of 7 coupled GaAs quantum wells (QWs). $\mathrm{Al}_{0.34} \mathrm{Ga}_{0.66} \mathrm{As}$ barriers are used in order to reach a conduction band-offset of $275 \mathrm{meV}$. N-doping of the first QW 
$\left(N_{d}=5 \times 10^{11} \mathrm{~cm}^{-2}\right)$ of each period allows to populate its first energy level $E_{1^{\prime}}$ in the conduction band with electrons. The layer sequence in $\AA$ starting from the first quantum well is as follows $68 / \mathbf{5 6 . 5} / 20 / \mathbf{3 9 . 5 5} / 23 / \mathbf{3 1} / 28 / \mathbf{3 1} / 34 / \mathbf{3 1} / 39 / \mathbf{3 1} / 48 / \mathbf{2 2 . 6}$ (the barriers are represented in bold types). Figure 1 recalls the principle of the device: owing to the absorption of a mid-infrared photon, an electron is excited from the fundamental level of the structure $E_{1^{\prime}}$ to the two excited states $E_{7}$ and $E_{8}$ which are delocalized across the first two QWs. High matrix elements between $E_{7 / 8}$ and other energy levels ( $E_{6}$ and $E_{5}$ in particular) allows the electron to be transferred to the right QWs as a result of a series of LO-phonon relaxations through the cascade of levels. The period is repeated in order to increase the induced potential that results from this electron transfer. By closing the circuit, a significant photocurrent is expected without any applied bias. The studied samples are $100 \times 100 \mu \mathrm{m}^{2}$ square mesas obtained by Reactive Ion Etching (RIE).

\section{MAGNETO-TRANSPORT MEASUREMENTS}

$R_{0} A$ is usually described with an activation energy $E_{a}$, corresponding to the energy of the main electronic transition in the structure. However, dark current $\left(I_{\text {dark }}\right)$ in QCDs often originates from several parallel cross transitions for a fixed temperature. As a result, a simple analysis taking into account one activation energy only does not accurately describe the system. In this context, our objective is to identify by magneto-transport measurements, the different transitions involved in $I_{\text {dark }}$ as a function of the temperature.

QCDs are mounted inside an insert at the center of a superconducting coil capable of a maximum field of $15 \mathrm{~T}$ such that the magnetic field lines are perpendicular to the QWs planes and parallel to the current flow direction: $I \| B$. The experiment consists of measuring $I_{\text {dark }}$ along the device, kept under constant voltage, while the magnetic field is swept up to its maximum value for three different temperatures $40 \mathrm{~K}, 80 \mathrm{~K}$ and $120 \mathrm{~K}$. Current measurements have been performed under a bias of $V=0.1 \mathrm{~V}$ for $T=80 \mathrm{~K}$ and $120 \mathrm{~K}$. At $40 \mathrm{~K}$ however, the current measured under $0.1 \mathrm{~V}$ was too low and results in a noisy curve. Therefore, a higher voltage of $1.5 \mathrm{~V}$ is applied to obtain a higher current value and a reliable measurement. A typical result is illustrated in figure 2(a) for a temperature of $120 \mathrm{~K}$. The dark current shows slight oscillations as a function of the magnetic field, superposed on a general behavior corresponding approximately to a quadratic decrease. The latter contribution can be accounted for by using a second order polynomial fit $a B^{2}+b B+c$. For example, a possible fit is found taking $a=-3.52 \times 10^{-6}, b=-2.86 \times 10^{-5}, c=5.75 \times 10^{-3}$. 
Then, this line shape is removed from the experimental $I_{\text {dark }}(B)$ curve and gives $\Delta I_{\text {dark }}$ shown in figure 2(b). This latter curve reveals a clear oscillating behavior which can be understood on the basis of the following arguments.

At zero magnetic field, all the quantum levels of a period have plane-wave-like energy dispersion in the direction parallel to the layers and all the corresponding energy subbands are nearly parallel (we do not consider the nonparabolicity effects which are negligible in GaAs for our energies $^{6,7}$ ). Most of electrons are localized in the first energy subband $E_{1^{\prime}}$. LO-phonon absorption and emission occur between this ground subband and several subbands of the neighboring cascade. At $0 \mathrm{~V}$, emission and absorption of LO phonon processes in $G_{i j}$ and $G_{j i}$ compensate each other resulting in zero current (the system is at equilibrium). With an applied bias, this equilibrium between emission and relaxation is broken and results in a dark current. When a magnetic field is applied, the subbands split into ladders of discrete Landau levels given by:

$$
E_{n, p}=E_{n}^{0}+\left(p+\frac{1}{2}\right) \hbar \omega_{c}
$$

where $n$ and $p$ are integers, $n$ is the index of the subband and $p$ index of the Landau level, $\hbar \omega_{c}=$ $\hbar e B / m^{\star}$ is the cyclotron energy, and $m^{\star}$, the effective mass in GaAs. $E_{n}^{0}$ is the energy of the subband edge at zero magnetic field. The effect of the magnetic field on the QCD dark current is quite similar to what happens in a three-level active region of a quantum cascade laser where electron-scattering from the upper state is modulated by magnetic field ${ }^{8}$. Indeed, depending on the value of the magnetic field, the Landau level arrangement strongly influences the absorption or emission of optical phonons from the various $\left|1^{\prime}, p\right\rangle$ levels to $|n, 0\rangle$, where $|n, p\rangle$ designates the $p$ Landau level originating from subband $n$. Figure 3 illustrates the effect of the Landau level quantization by a magnetic field on the dark current taking as example level $|6,0\rangle$. Electrons are mainly localized in the fundamental level $E_{1^{\prime}}$ and are distributed over the different Landau levels $\left|1^{\prime}, p\right\rangle$ according to a Fermi-Dirac distribution. At $8.8 \mathrm{~T}$, considering a temperature such that $\left|1^{\prime}, 5\right\rangle$ is populated, LO-phonon absorption from $\left|1^{\prime}, 5\right\rangle$ to $|6,0\rangle$ is inhibited because this process does not conserve the energy. For a higher value of the magnetic field $(9.6 \mathrm{~T})$, levels $\left|1^{\prime}, 5\right\rangle$ and $|6,0\rangle$ are separated by the exact energy of a LO-phonon ( $\hbar \omega_{L O}=36 \mathrm{meV}$ in GaAs), permitting LOphonon absorption or emission: electrons can short-circuit the cascade generating a dark current. Dark current shows a maximum whenever an electron on the $\left|1^{\prime}, p\right\rangle$ level can be excited to a $|n, 0\rangle$ Landau level by absorption of one LO-phonon. This is called a magneto-phonon resonance (MPR) and appear as oscillations of the dark current as a function of the magnetic field. Maxima of these 
oscillations correspond to a Landau level $p$ in resonance with the upper state minus $\hbar \omega_{L O}$. On figure 2(a), we can see that these oscillations only begin from $5 \mathrm{~T}$. As Landau levels are broadened by disorder, their finesse increase as the square root of the magnetic field. Therefore, at high magnetic fields, the related Landau level becomes increasingly populated, increasing the effect of MPRs. For a fixed temperature, several transitions $1^{\prime} \rightarrow n$ participate simultaneously to dark current such that each transition generates its own series of oscillations. Considering a transition from $\left|1^{\prime}, p\right\rangle$ to $|n, 0\rangle$, values of magnetic field $B_{1^{\prime}, p}$, which give rise to resonant optical-phonon absorption, satisfy the following equation:

$$
E_{n, 0}-E_{1^{\prime}, p}\left(B_{1^{\prime}, p}\right)=\hbar \omega_{L O}
$$

or

$$
\Delta E_{n-1}^{0}-\hbar \omega_{L O}=\frac{p \hbar e B_{1^{\prime}, p}}{m^{\star}}
$$

where $\Delta E_{n-1}^{0}$ is the energy separation between subband 1 and $n$ at zero magnetic field. Oscillations appear periodically as a function of the inverse of the magnetic field, the period of which is given by:

$$
\frac{1}{B_{1^{\prime}, p-1}}-\frac{1}{B_{1^{\prime}, p}}=\frac{\hbar e}{m^{\star}\left(\Delta E_{n-1}^{0}-\hbar \omega_{L O}\right)}
$$

The measurement of $I_{\text {dark }}\left(V, B^{-1}\right)$ allows us to determine $\Delta E_{n-1}^{0}$. However, as $I_{\text {dark }}\left(V, B^{-1}\right)$ results from the superposition of several series of oscillations, it remains difficult to identify clearly the periodicity relative to each contributing transition. For this purpose, we performed a Fourier transform of the corresponding $\Delta I_{\text {dark }}\left(V, B^{-1}\right)$ curves in order to extract the characteristic frequencies of each oscillation series.

Since the working temperature of our QCD is around $80 \mathrm{~K}$, we first show the experimental results at this temperature (figure 4): this is the most important result as far as the device is concerned. For a full understanding of the system, it is also very interesting to show the same results at $40 \mathrm{~K}$ and $120 \mathrm{~K}$ (figure 5 and 6, respectively).

In these figures, the Fourier transform of the dark current measurements are reported. To put in evidence the energy of the electronic transitions, the Fourier transform amplitude is plotted as a function of $\Delta E$ (through $\left.\Delta E=\hbar e B / m^{\star}+\hbar \omega_{L O}\right)$ rather than with the initial $B$-scale. The inset is a guide for the eye to identify the electronic transitions observed in these spectra. Table I provides the global transition rates $G_{1^{\prime} j}$, i.e. the number of transitions per second and per square meter from the fundamental level $1^{\prime}$ to the level $j$, with $j=\{2, \ldots, 8\}$, in the neighboring cascade. (see Part 
IV) For each temperature, the experimental results extracted from each spectrum can be faced to these theoretical calculations.

At $80 \mathrm{~K}$ (figure 4), six peaks can be identified. The peaks at 52, 64, 83, 118 and $145 \mathrm{meV}$ correspond to LO-phonon assisted cross transitions from the fundamental subband $1^{\prime}$ to subbands $3,4,5,6$ and $7 / 8$ respectively. These peaks are well explained by the model since the calculation shows significant transition rates, $G_{1^{\prime} j}$. The last peak, at around $180 \mathrm{meV}$, is attributed to phonon absorption from subband $2^{\prime}$ to a quasi-bound state $E_{9^{\prime}}$ situated at the border of the conduction band. This transition is possible because the subband $E_{2^{\prime}}$ is not totally empty and the overlap between these two states $E_{2^{\prime}}$ and $E_{9^{\prime}}$ is significant. We can notice that the two first peaks corresponding to phonon absorption from $E_{1^{\prime}}$ to $E_{3}$ and $E_{4}$ have high amplitudes. These amplitudes are fairly large, in contradiction with the quantitative values of $G_{1^{\prime} j}$. We attribute this effect to all the electron relaxations in the cascade that give also rise to oscillation series in the same energy range. As a result, a quantitative study in this energy range is not accessible for the moment where intercascade resonances are mixed with intracascade resonances and/or elastic scattering. At higher energies, the model provides quasi equal global transition rates for transitions $E_{1^{\prime}}$ to $E_{5}, E_{6}$ and $E_{7 / 8}$, in good accordance with the amplitude of the relative peaks in the spectra. These measurements show that the magnetic field is a powerful spectroscopic tool to probe the different transitions responsible for dark current in QCDs.

At $40 \mathrm{~K}$ (figure 5), we identify four peaks. Two dominant peaks at $49 \mathrm{meV}$ and $63.5 \mathrm{meV}$ originate from the oscillation series associated with absorption of a LO-phonon from $E_{1^{\prime}}$ to $E_{3}$ and $E_{1^{\prime}}$ to $E_{4}$, respectively. Likewise at higher energies the curve exhibits two peaks at $85 \mathrm{meV}$ and $120 \mathrm{meV}$ which represent $E_{1^{\prime}} \rightarrow E_{5}$ and $E_{1^{\prime}} \rightarrow E_{6}$, respectively. For the same reason as before a quantitative comparison of the amplitude of these peaks is not accessible.

Finally, at $120 \mathrm{~K}$ (figure 6), we observe the disappearance of the transition $E_{1^{\prime}} \rightarrow E_{6}$ and the parallel enhancement of both transitions $E_{1^{\prime}} \rightarrow E_{7 / 8}$ and $E_{2^{\prime}} \rightarrow E_{9^{\prime}}$. These results are in excellent agreement with the calculations of the model providing two equal global transition rates for transitions $E_{1^{\prime}} \rightarrow E_{7}$ and $E_{1^{\prime}} \rightarrow E_{8}$ (see table I). We can add that a non negligible quantity of electrons are now present in level $9^{\prime}$ at high energy: relaxations of electrons from this last subband will give rise to a higher energy peak in the spectrum that is present in figure 6 from 165 to $200 \mathrm{meV}$. Note that at this high temperature, the intracascade transitions and/or elastic scattering give a wide peak at low energy.

We have shown that the transitions contributing to the dark current are highly temperature 
dependent. These results can be understood by the fact that dark current is a result of a compromise between the temperature-dependant number of electrons available at a certain energy $E_{j}-\hbar \omega_{L O}$ and the matrix element between the fundamental subband $E_{1^{\prime}}$ and $E_{j}$ in the neighboring cascade. $G_{1^{\prime} j}$ is proportional to both, for the former preventing high $j E_{j}$-paths at low temperature and for the latter, decreasing the effect for low $j E_{j}$-paths owing to the low spatial overlap between $E_{1^{\prime}}$ and $E_{j}$. This explanation appears very clearly in the expression of the global transition rate $G_{i j}^{a}$ (the superscript " $a$ " means that this rate describes only phonon absorption mediated transitions from subband $i$ to subband $j)^{9}$ :

$$
G_{i j}^{a}=\int_{E_{j}-\hbar \omega_{L O}}^{+\infty} S_{i j}^{a}(E) f(E)\left(1-f\left(E+\hbar \omega_{L O}\right)\right) n_{\text {opt }} \times \mathcal{D}(E) d E
$$

where $S_{i j}^{a}$ is the electron-LO phonon transition rate ${ }^{10}, f(E)$ and $f\left(E+\hbar \omega_{L O}\right)$ are the Fermi-Dirac occupation factor at $E$ and $E+\hbar \omega_{L O}, \mathcal{D}(E)$ is the two-dimensional density of state of the subband $j$ and $n_{o p t}$ is the Bose-Einstein statistic function which accounts for the phonon population. In this expression, the compromise emerges from the matrix element $S_{i j}$ and the two terms $f(E)$ and $f\left(E+\hbar \omega_{L O}\right)$ that give approximately the electron density in the subbands $i$ and $j$.

To conclude, as well as establishment of the different transitions involved in the transport, their importance as a function of temperature is also demonstrated: at low temperature, the very low occupation factor of high energy levels forbids their participation to the transport. At higher temperature (more than $100 \mathrm{~K}$ ), these high energy levels dominate the transport thanks to their high matrix element with $E_{1^{\prime}}$. The most interesting situation is at $80 \mathrm{~K}$, as it is the optimal temperature for a focal plane array at $8 \mu \mathrm{m}$ wavelength. At $80 \mathrm{~K}$, the different transitions $E_{1^{\prime}} \rightarrow E_{j}$ seem to have similar importance, and are all identified. The "rule of thumb" for the design of a QCD, as detailed in the introduction is found again: at this working temperature, $G_{1^{\prime} j}$ for $j \leq 6$ should be slightly lower or equal to the $G_{1^{\prime} 7}$ and $G_{1^{\prime} 8}$. To put accurate numbers on these quantities, part IV concentrates on the modeling of these global transition rates and of the current.

\section{THEORETICAL MODEL OF DARK TRANSPORT IN QCDS}

In this part, we will describe in detail the model that leads to the $G_{1^{\prime} j}$ shown in table I, and to the $I(V)$ curve of the QCDs. This model is able, without any adjustable parameter, to give an excellent value of the resistance of a QCD. Starting from the well-known electron-LO phonon transition rate $^{8}$, this model is based on an analogy with the p-n junction, an archetype system where 
two reservoirs (the conduction and valence band) are separated by a conduction bottleneck. In our case, it has been shown that intra-cascade global transition rates are several orders of magnitude higher than inter-cascade global transition rates such that two neighboring cascades $(A$ and $B)$ act as two reservoirs separated by a bottleneck. As a result, in the same way as in a p-n junction, quasi-Fermi levels can be associated with each cascade and, starting from this hypothesis, a simple expression can be derived for the resistance of the device at zero bias:

$$
R_{0} A=\frac{k_{B} T}{q^{2} \sum_{i \in A} \sum_{j \in B} G_{i j}}
$$

where $T$ is the temperature and the term $G_{i j}$ is defined by the sum of $G_{i j}^{a}$ and $G_{i j}^{e}$ calculated at equilibrium i.e. without any applied voltage (in $G_{i j}^{a}$ and $G_{i j}^{e}$, the superscript " $a$ " or " $e$ " stands for phonon absorption or emission, respectively). This expression of $R_{0} A$ as a function of $\sum_{i \in A} \sum_{j \in B} G_{i j}$ results from a calculation of the current from the electrons going through an imaginary surface separating the two reservoirs $A$ and $B$. The detailed derivation of (7) can be found in ref $^{9}$. Here, instead of the classical $R_{0} A$ parameter, we will present the results through the conductance $G_{0}$ which is directly proportional to the current through the device:

$$
\frac{G_{0}}{A}=\frac{1}{R_{0} A}=\frac{q^{2}}{k_{B} T} \times \sum_{i \in A} \sum_{j \in B} G_{i j}
$$

According to this expression, transitions appear very clearly as many parallel paths for electrons to join cascade $B$ from cascade $A$. Expression (8) can also be seen as an Einstein relation, linking a macroscopic transport property $G_{0}$ to microscopic diffusion coefficients $G_{i j}$. This relation is expected since the transport is described as a diffusion process resulting from a non-homogeneous chemical potential. This is indeed the case in QCDs where the current is calculated as a result of a variation of the distribution of carriers as a function of the energy from cascade $A$ to cascade $B$.

In figure 7 , the experimental $G_{0} / A$ is represented with circles and compared to $q^{2} G_{1^{\prime} j} / k_{B} T$, where $j=\{2, \ldots, 8\}$ as a function of $1000 / T$. This figure shows very clearly the progressive implication of the different transitions to the dark current as a function of the temperature: at $300 \mathrm{~K}(1000 / T=3.33)$, as expected, the two curves corresponding to $G_{1^{\prime} 8}$ and $G_{1^{\prime} 7}$ are much closer to the experimental conductance. Around $80 \mathrm{~K}$, dark current implicates the quasi totality of the diagonal transitions. This is an illustration of the rule of thumb for the design of a QCD, as discussed previously, all the transitions $G_{1^{\prime} j}$ show the same order of magnitude, in order to optimize the trade-off between a high escape probability in the cascade, a high optical coupling 
between $E_{1^{\prime}}$ and $E_{7}$, and a low leakage between $E_{1^{\prime}}$ and $E_{j}, j \leq 6$. As shown in table I, $G_{1^{\prime} 5}$ and $G_{1^{\prime} 6}$ are slightly greater than $G_{1^{\prime} 7}$ and $G_{1^{\prime} 8}$ at $80 \mathrm{~K}$, which can be understood in two ways: either this detector is considered as too noisy for a working temperature of $80 \mathrm{~K}$, or it is much more suited for working at higher working temperature. Finally, at $40 \mathrm{~K}, G_{0} / A$ is no more close to $G_{i j} / A$, as the resistance in the cascade has now to be taken into account and our model, which only considers diagonal transitions, becomes invalid as the two cascades are no longer in quasiequilibrium. Another discrepancy between the model and the experiment at low temperature is that we have considered only the optical phonon interaction to transfer the electrons from one subband to another ${ }^{11-13}$. Other interactions such as interface roughness scattering can become dominant at low temperature, as shown by A. Leuliet et al. ${ }^{14}$ Other interactions such as impurities scattering and alloy scattering can also play a significant role.

Figure 8 compares the calculated (triangles) and measured (circles) $G_{0} / A$ : the agreement is excellent over five orders of magnitude from $300 \mathrm{~K}$ to $80 \mathrm{~K}$. This validates the assumptions that have been made at the basis of this model: the restriction of the transfer mechanisms to the electron-optical phonon interaction (acoustical phonons, electron-electron interactions, interface roughness scattering, have been neglected), and quasi equilibrium hypothesis inside a cascade. Nevertheless, at lower temperatures, a difference of about one or two order of magnitude exists between the calculated and experimental conductance. In this range of temperature, transitions inside the cascade are limiting the dark current and now need to be taken into account to completely describe the electronic dark transport. The hypothesis of two quasi Fermi levels fails and the determination of the voltage drop at each point of the structure is necessary to calculate the current. Such a complex calculation is beyond the scope of this paper and also not necessary as far as infrared detection is concerned, since thermal imagers do not work at such low temperatures.

We can now complete these results by a comparison between the activation energy extracted from the experimental and calculated $R_{0} A$ (see figure 9) given by slope of the logarithm of the $R_{0} A$ as a function of $1000 / T$. From room temperature down to $120 \mathrm{~K}$, the two curves exhibit the same activation energy of $120 \mathrm{meV}$. Magneto-transport measurements have shown that dark current also implicates higher energy transitions $\left(E_{1^{\prime}} \rightarrow E_{7 / 8}\right.$ and $\left.E_{2^{\prime}} \rightarrow E_{9^{\prime}}\right)$. This complexity is hidden behind a single resistance measurement; this shows the power of magneto transport measurements. At lower temperature, a discrepancy appears: at $80 \mathrm{~K}$, the experimental activation energy is first higher (about $110 \mathrm{meV}$ ) than the energy given by the theoretical predictions of the model $(80 \mathrm{meV})$. Finally, at $40 \mathrm{~K}$, the calculated activation energy of $46 \mathrm{meV}$ (transition $E_{1^{\prime}} \rightarrow$ 
$E_{3}$ ) is very different from the experimental value of $16-17 \mathrm{meV}$ corresponding typically to the energy of a transition inside the cascade showing again that, at low temperature, the hypothesis of two separated cascades at quasi-equilibrium fails.

In conclusion, two different regimes in the dark transport are observed: from $300 \mathrm{~K}$ to $100 \mathrm{~K}$, dark transport is dominated by cross transitions that is in good agreement with the model. The model considers that the electronic displacement inside a cascade is several orders of magnitude faster than between two consecutive cascades. In the second regime, at lower temperatures, relaxations in the cascade are less efficient. In particular, electrons are mostly located around $k=0$ and cannot relax easily to subbands separated by less than the energy of a LO-phonon $\left(E_{4}-E_{3}=17 \mathrm{meV}\right.$, for example).

Up to now, we have shown that our model can be used to calculate the $R_{0} A$ parameter in order to predict the Johnson noise in photovoltaic QCDs at $0 \mathrm{~V}$. However, the full $I(V)$ of the device is also interesting as in some cases, working with a small applied bias can become an advantage for example in a camera. As this kind of device always presents a high non-linear resistance, it is essential to know the value of the current along the QCD in order to adapt the applied bias to the capacity of the read-out circuit, the detector and the cameras' characteristics (f number, background temperature, integration time).

As explained before, the global current density is evaluated by counting the electronic transitions between two consecutive cascades $A$ and $B$. The global current density is given by equation (1). In equation (1), $G_{i j}(V)$ is the sum of two global transition rates, one for LO-phonon absorption and one for the LO-phonon emission. The current density $J$ is given by:

$$
J=q \sum_{i \in A} \sum_{j \in B}\left(G_{i j}^{a}(V)-G_{j i}^{e}(V)+G_{i j}^{e}(V)-G_{j i}^{a}(V)\right)
$$

The difference $G_{i j}^{a}(V)-G_{j i}^{e}(V)$ can be expressed as:

$$
\begin{gathered}
G_{i j}^{a}(V)-G_{j i}^{e}(V)=\int_{E_{j}-\hbar \omega_{L O}}^{+\infty} S_{i j}^{a}(E) n_{\text {opt }} f_{A}(E)\left[1-f_{B}\left(E+\hbar \omega_{L O}\right)\right] \mathcal{D}(E) d E \times[1-\gamma(E)] \\
\gamma(E)=\frac{f_{B}\left(E+\hbar \omega_{L O}\right)\left(1-f_{A}(E)\right)\left(1+n_{\text {opt }}\right)}{f_{A}(E)\left(1-f_{B}\left(E+\hbar \omega_{L O}\right)\right) n_{\text {opt }}}
\end{gathered}
$$

In QCDs, as in many other photovoltaic detectors, the applied voltage is small. In this case, we can assimilate $\alpha(E)=n_{\text {opt }} f_{A}(E)\left(1-f_{B}\left(E+\hbar \omega_{L O}\right)\right) \mathcal{D}(E)$ to its value at equilibrium given 
by $\alpha_{e q}(E)=n_{\text {opt }} f(E)\left(1-f\left(E+\hbar \omega_{L O}\right)\right) \mathcal{D}(E)$. Expressing the Fermi-Dirac function, $\gamma(E)$ is simplified to:

$$
\gamma(E)=\exp \left(\frac{E_{F}^{B}-E_{F}^{A}}{k_{B} T}\right)=\exp \left(\frac{-q V}{k_{B} T}\right)
$$

leading us to a final expression:

$G_{i j}^{a}(V)-G_{j i}^{e}(V)=\int_{E_{j}-\hbar \omega_{L O}}^{+\infty} S_{i j}^{a}(E) \alpha_{e q}(E) d E \times\left[1-\exp \left(\frac{-q V}{k_{B} T}\right)\right]=G_{i j}^{a}(0) \times\left[1-\exp \left(\frac{-q V}{k_{B} T}\right)\right]$

The contribution of $G_{i j}^{a}-G_{j i}^{e}$ to $G_{0} / A$ can be simply expressed as $G_{i j}^{a}(0) q / k_{B} T$, and the nonlinear resistance or asymmetry in $I(V)$ characteristic is furthermore included in the simple exponential term. In this approximation, the QCD shows a standard diode behavior with an ideality factor equal to 1 . We find a similar expression for $G_{i j}^{e}(V)-G_{j i}^{a}(V)$ :

$$
G_{i j}^{e}(V)-G_{j i}^{a}(V)=G_{i j}^{e}(0) \times\left[1-\exp \left(\frac{-q V}{k_{B} T}\right)\right]
$$

Summing the two last expressions, the total current density is finally given by:

$$
J=j_{0}\left[1-\exp \left(\frac{-q V}{k_{B} T}\right)\right]
$$

with

$$
j_{0}=q \sum_{i \in A} \sum_{j \in B}\left(G_{i j}^{a}(0)+G_{i j}^{e}(0)\right)=q G_{t o t}
$$

Figure 10 and 11 represent the experimental (circles) and calculated (triangles) $I(V)$ characteristic for a $100 \mu \mathrm{m} \times 100 \mu \mathrm{m}$ area pixel at $120 \mathrm{~K}, 80 \mathrm{~K}$, respectively. At $120 \mathrm{~K}$, using the global transition rate $G_{t o t}=7.66 \times 10^{21} \mathrm{~m}^{-2} \mathrm{~s}^{-1}$ provided by the model, the agreement between the experimental and calculated current is excellent over a voltage range of $\pm 0.1 \mathrm{~V}$. Let us recall that this model requires no adjustable parameter other than the doping density. At $80 \mathrm{~K}$, a discrepancy appears between the calculated and experimental curve (figure 11). This divergence was expected considering the disagreement between the experimental and calculated $G_{0} / A$ (a factor 2.3 between the two). (A perfect agreement can be obtained using a global transition rate of $1.47 \times 10^{19} \mathrm{~m}^{-2} \mathrm{~s}^{-1}$ ). At $40 \mathrm{~K}$, the calculations disagree with the experimental $I-V$ characteristics because, the dark current is now limited by electronic relaxations within the cascade (see above). 


\section{CONCLUSION}

In this paper, magneto-transport experiments have been performed on quantum cascade detectors. In these complex multiple quantum wells heterostructures, the transport is shown to result from electronic diffusion events from subbands to subbands. Many different electronic paths are involved in the transport as different parallel channels. Magneto-transport experiments give a unique possibility to highlight these different paths, showing separately the intersubband electron transitions significantly involved in the transport. In particular, it is shown that low energy transitions are preferred at low temperature, while higher energy transitions participate at higher temperatures, where the electronic population in the final subband is thermally activated. This shows that the conception of a QCD should include the final working temperature as a crucial quantum design parameter.

A model describing the transport in quantum cascade structures has also been developed. It relies on the modeling of the current as a diffusion from two reservoirs at quasi equilibrium (cascades $A$ and $B$ ), separated by a bottleneck. To calculate the transfer rates between the subbands, a simple perturbative approach has been developed (where they are expressed as a function of the transfer rate at $0 \mathrm{~V}$ ) considering the electron-optical phonon interaction only. The model and the important assumptions have been validated by several experimental results: magneto transport, $R_{0} A$ and $I(V)$ curves. $R_{0} A$ as a function of the temperature is fitted by our model with an excellent approximation of more than 5 orders of magnitude down to $\sim 100 \mathrm{~K}$, with only the doping density as an adjustable parameter. Furthermore, the $I(V)$ curve of the diode, which is important for detector integration with a read-out circuit, can also be predicted with the model, within an excellent agreement also down to $100 \mathrm{~K}$ (for our example of a QCD with an optical transition at $155 \mathrm{meV})$. The agreement begins to fail at lower temperature.

This kind of model is a crucial tool for the conception of a QCD structure. The QCD is the first detector which can be completely realized and optimized without the use of phenomenological parameter due to the fact that in a QCD, electrons only occupy two dimensional states, allowing the realization of a robust model. This is in strong contrast to other detectors such as QWIPs where 3D states in the continuum result in complications such as capture and escape probabilities. These are difficult to calculate and therefore considered as adjustable parameters that have been determined experimentally during the past 15 years. 


\section{ACKNOWLEDGMENTS}

This work has been supported by the French DGA and by a grant of the Agence Nationale pour la Recherche (ANR). The authors are indebted to S. Dhillon for a critical reading of the manuscript.

* Electronic address: vincent.berger@univ-paris-diderot.fr

1 L. Gendron, M. Carras, A. Huynh, V. Ortiz, C. Koeniguer, and V. Berger, App. Phys. Lett. 85, 2824 (2004)

2 L. Gendron, C. Koeniguer, and V. Berger, Appl. Phys. Lett. 86, 121116 (2005)

3 M. Graf, G. Scalari, D. Hofstetter, J. Faist, H. Beere, E. Linfield, D. Ritchie, and, G. Davies, App. Phys. Lett. 84, 475 (2004)

4 G. Scalari, M. Graf, D. Hofstetter, J. Faist, H. Beere, and D. Ritchie, Semicond. Sci. Technol. 21, $1743-$ $1746(2006)$

5 B. F. Levine, J. Appl. Phys. 74, R1-R81 (1993)

6 I. Vurgaftman, J.R. Meyer, and L.R. Ram-Mohan, J. Appl. Phys. 89, 5815 (2001)

7 C. Sirtori, F. Capasso, J. Faist, and S. Scandolo, Phys. Rev. B 50, 8663 (1994)

8 D. Smirnov, C. Becker, O. Drachenko, V.V. Rylkov, H. Page, J. Leotin, and C. Sirtori, Phys. Rev. B 66, 121305(R) (2002)

9 C. Koeniguer, G. Dubois, A. Gomez, and V. Berger, Phys. Rev. B 74, 235325 (2006)

10 R. Ferreira, G. Bastard, Phys. Rev. B 40, 1074 (1989)

11 J.A. Brum and G. Bastard, Phys. Rev. B 33, 1420 (1986)

12 K.Kempa, Y. Zhou, J.R. Engelbrecht, P. Bakshi, H.I. Ha, J. Moser, M.J. Naughton, J. Ulrich, G. Strasser, E. Gornik, and K. Unterrainer, Phys. Rev. Lett. 88, 226803 (2002)

13 K.Kempa, Y. Zhou, J.R. Engelbrecht, and P. Bakshi, Phys. Rev. B 68, 085302 (2003)

14 A. Leuliet, A. Vasanelli, A. Wade, G. Fedorov, D. Smirnov, G. Bastard, and C. Sirtori, Phys. Rev. B 73, $085311(2006)$ 


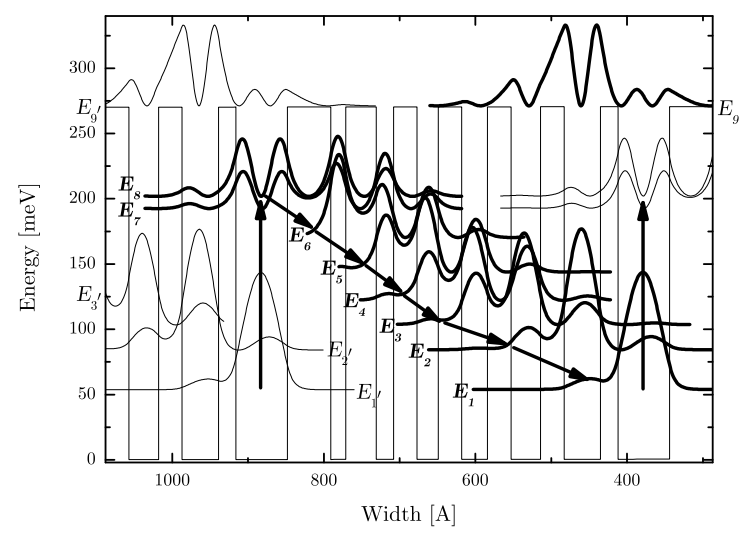

FIG. 1: Conduction band diagram of one period of an $8 \mu \mathrm{m}$ QCD showing the energy levels. Note that the ground state of the first QW belongs to the former period and is noted $E_{1^{\prime}}$. The arrows illustrate the electronic path during a detection event.

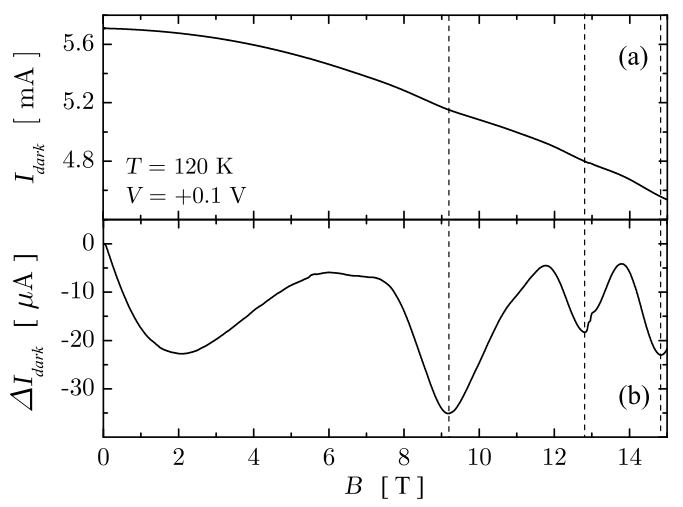

FIG. 2: (a) Dark current as a function of the magnetic field for a fixed value of the voltage $0.1 \mathrm{~V}$ at $120 \mathrm{~K}$. (b) Dark current as a function of the magnetic field where the contribution of the magneto-resistance of the contacts has been subtracted. 


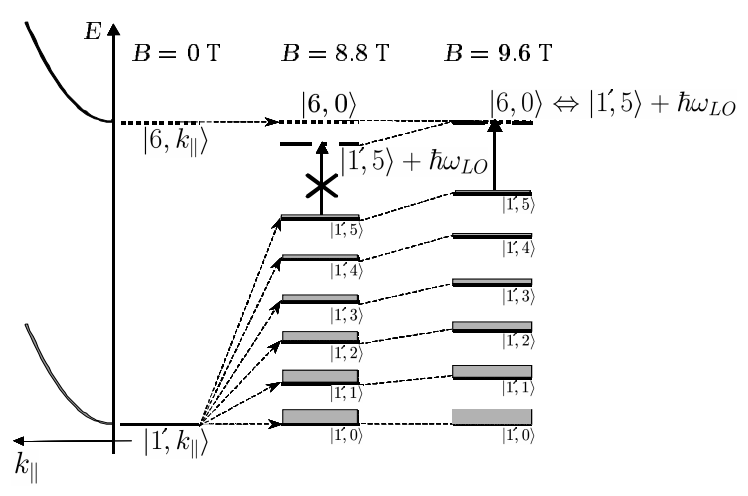

FIG. 3: Schematic of the fundamental energy level $E_{1^{\prime}}$ and the level $E_{6}$ at $B=0 \mathrm{~T}$ and for two different magnetic fields. At $B=0 \mathrm{~T}, E_{1^{\prime}}$ and $E_{6}$ have free particle-like dispersion in the direction parallel to the layers $E=\hbar^{2} k_{\|}^{2} / 2 m^{\star}$ where $k_{\|}$is the corresponding wave number. The magnetic field breaks the subbands into two ladders of Landau levels, represented by horizontal segments (solid line for landau ladder originating from the fundamental $n=1^{\prime}$ state and dotted line for the $n=6$ state). The black arrows represent LO-phonon absorption, allowed for $B=9.6 \mathrm{~T}$ but forbidden for $8.8 \mathrm{~T}$. For sake of clarity, all Landau levels have been lowered by $\hbar e B / 2 m^{\star}$.

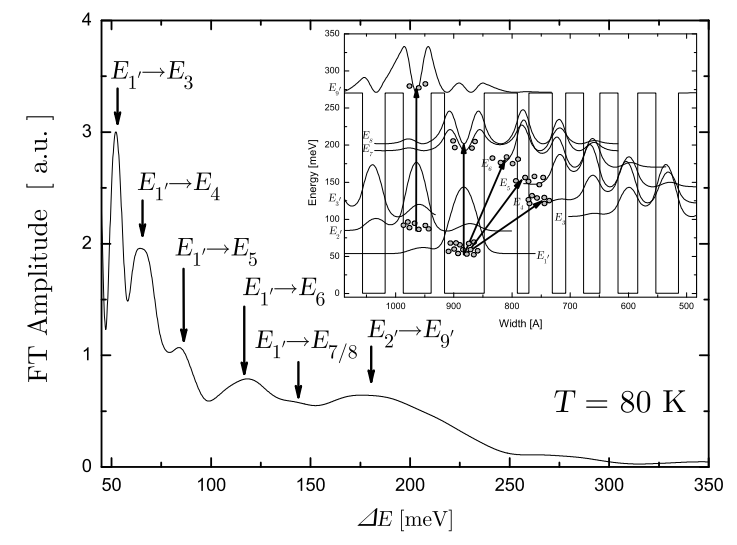

FIG. 4: Fourier Transform amplitude of experimental $\Delta I_{\text {dark }}$ vs $\Delta E=\hbar e B / m^{\star}+\hbar \omega_{L O}$ at $80 \mathrm{~K}$. 


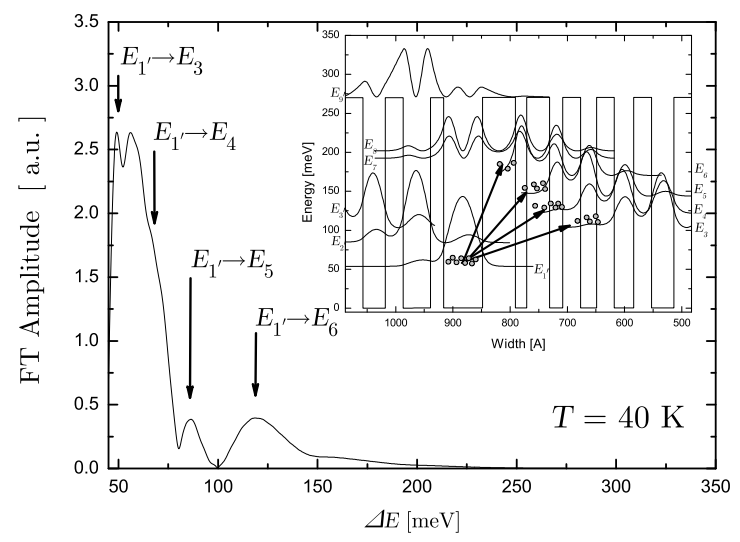

FIG. 5: Fourier Transform amplitude of experimental $\Delta I_{\text {dark }}$ vs $\Delta E=\hbar e B / m^{\star}+\hbar \omega_{L O}$ at $40 \mathrm{~K}$.

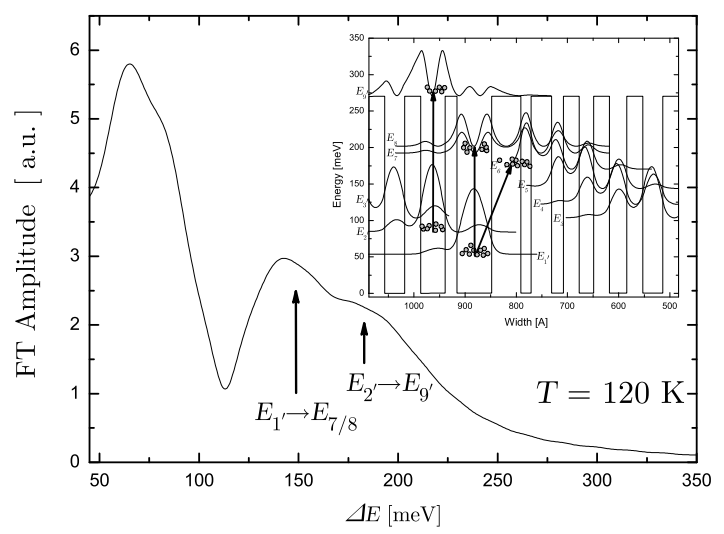

FIG. 6: Fourier Transform amplitude of experimental $\Delta I_{\text {dark }}$ vs $\Delta E=\hbar e B / m^{\star}+\hbar \omega_{L O}$ at $120 \mathrm{~K}$. 


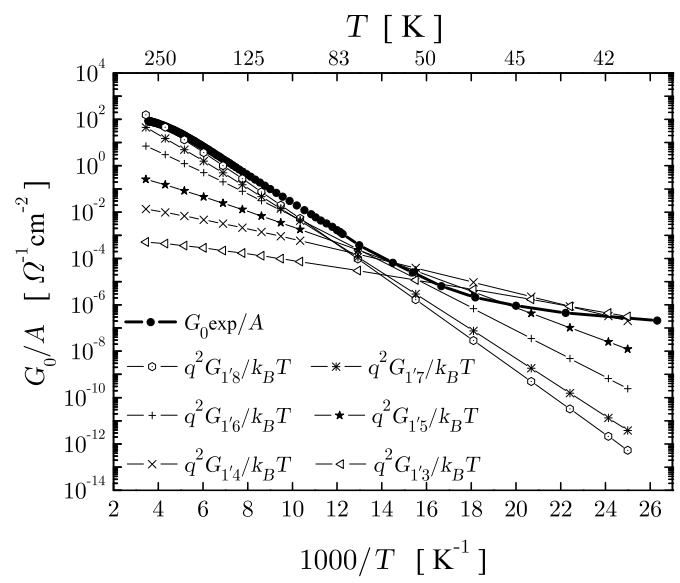

FIG. 7: $G_{0} / A$ as a function of $1000 / T$, where $T$ is the temperature of the sample. Solid line with circles corresponds to the experimental value while solid lines with symbols are the calculated $q^{2} G_{1^{\prime} j} / k_{B} T$ for $j=\{3, \ldots, 8\}$

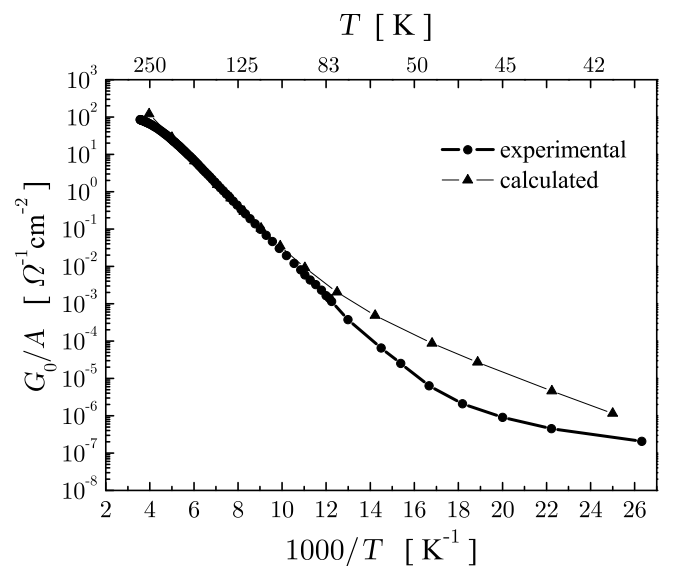

FIG. 8: $G_{0} / A$ as a function of $1000 / T$, where $T$ is the temperature of the sample. Solid line with circles corresponds to the experimental value, solid line with triangles is the calculated value $\left(q^{2} \sum_{i=1}^{8} \sum_{j=1}^{8} G_{i j} / k_{B} T\right)$ provided by the model. 


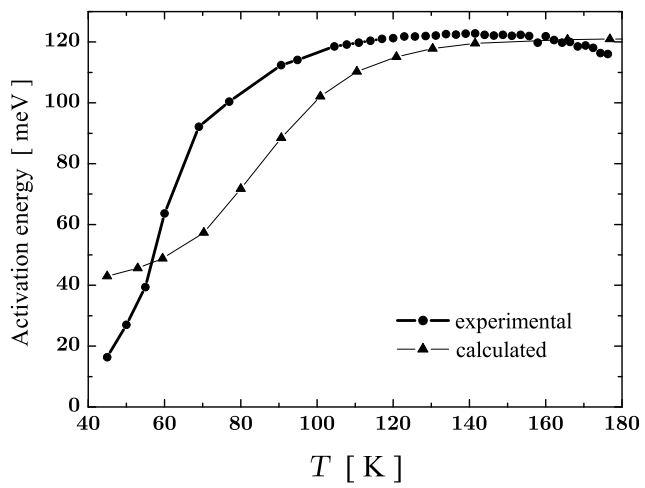

FIG. 9: Calculated (triangles) and experimental (circles) activation energy as a function of the temperature.

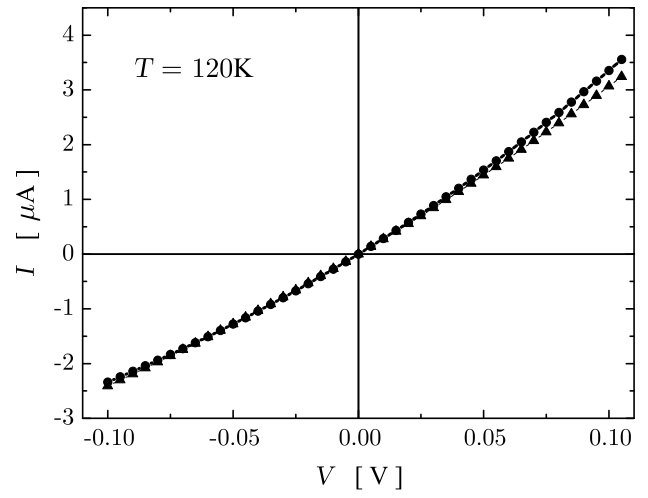

FIG. 10: Calculated (triangles) $\left(G_{t o t}=7.66 \times 10^{21} \mathrm{~m}^{2} \mathrm{~s}^{-1}\right)$ and experimental (circles) $I(V)$ characteristics for a $100 \mu \mathrm{m}$ pixel at $120 \mathrm{~K}$.

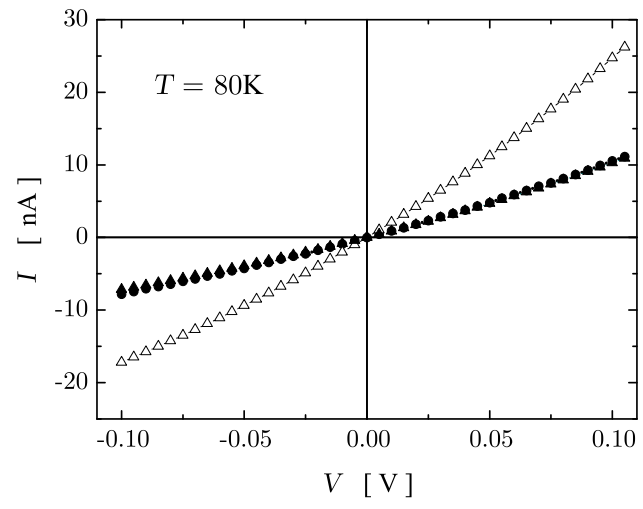

FIG. 11: Calculated (bold triangles correspond to $G_{t o t}=3.56 \times 10^{19} \mathrm{~m}^{2} \mathrm{~s}^{-1}$ and open triangles to $G_{t o t}=$ $1.47 \times 10^{19} \mathrm{~m}^{2} \mathrm{~s}^{-1}$ ) and experimental (circles) $I(V)$ characteristics for a $100 \mu \mathrm{m}$ pixel at $80 \mathrm{~K}$. 


\begin{tabular}{cccc}
\hline$G_{1^{\prime} j}\left(\mathrm{~m}^{-2} \mathrm{~s}^{-1}\right)$ & $40 \mathrm{~K}$ & $80 \mathrm{~K}$ & $120 \mathrm{~K}$ \\
\hline $1^{\prime} \rightarrow 2$ & $3.72 \times 10^{14}$ & $6.40 \times 10^{16}$ & $3.44 \times 10^{17}$ \\
$1^{\prime} \rightarrow 3$ & $\mathbf{3 . 1 6} \times \mathbf{1 0}^{\mathbf{1 5}}$ & $6.76 \times 10^{17}$ & $4.19 \times 10^{18}$ \\
$1^{\prime} \rightarrow 4$ & $\mathbf{1 . 5 2} \times \mathbf{1 0}^{\mathbf{1 5}}$ & $\mathbf{3 . 0 1} \times \mathbf{1 0}^{\mathbf{1 8}}$ & $3.93 \times 10^{19}$ \\
$1^{\prime} \rightarrow 5$ & $9.18 \times 10^{13}$ & $\mathbf{5 . 1 0} \times \mathbf{1 0}^{\mathbf{1 8}}$ & $2.09 \times 10^{20}$ \\
$1^{\prime} \rightarrow 6$ & $1.32 \times 10^{12}$ & $\mathbf{5 . 0 3} \times \mathbf{1 0}^{\mathbf{1 8}}$ & $8.72 \times 10^{20}$ \\
$1^{\prime} \rightarrow 7$ & $2.56 \times 10^{10}$ & $\mathbf{3 . 4 9} \times \mathbf{1 0}^{\mathbf{1 8}}$ & $\mathbf{2 . 0 3} \times \mathbf{1 0}^{\mathbf{2 1}}$ \\
$1^{\prime} \rightarrow 8$ & $2.90 \times 10^{9}$ & $\mathbf{2 . 3 2} \times \mathbf{1 0}^{\mathbf{1 8}}$ & $\mathbf{2 . 4 6} \times \mathbf{1 0}^{\mathbf{2 1}}$ \\
\hline
\end{tabular}

TABLE I: Values of the global transition rates $G_{1^{\prime} j}$ for $j=\{2, \ldots, 8\}$ at 40,80 and $120 \mathrm{~K}$. Bold characters correspond to the highest values of the global transition rates for each temperature. 